\title{
EU Floods Directive implementation in Austria
}

\author{
Clemens Neuhold ${ }^{1, \text { a }}$ \\ ${ }^{1}$ Federal Ministry of Agriculture, Forestry, Environment and Water Management, Vienna, Austria
}

\begin{abstract}
Floods have the potential to cause fatalities, displacement of people and damage to the environment, to severely compromise economic development and to undermine the economic activities of the Community. The EU Directive on the assessment and management of flood risks [2007/60/EC] was adopted on 23 October 2007. Its aim is to reduce and manage the risks that floods pose to human health, the environment, cultural heritage and economic activity. The paper reflects on how the requirements of the FD had been achieved in Austria and how the nationwide comparability and transferability of results as well as the international coordination had been obtained. Austria as a federal state has its competences structured in different departments as well as administrational levels. Besides administrational characteristics there is also a high diversity in topographical boundary conditions from Alpine areas to lowland areas emphasising different approaches and foci of flood risk management. To harmonise the related interests a discussion and decision committee had been established. The resolutions of this committee then defined the basis for a national coordination procedure where the Federal Ministry provided a "federal blueprint" to the federal provinces. The federal provinces then incorporated their regional and local information and data. Based on this response the coordinated and nationwide comparable FRMP had been set up and had been forwarded to public information and consultation. Complementary stakeholder involvement has been ensured by information and discussion workshops throughout the entire process. The administrational and topographical characteristics to be considered in the frame of FD implementation strengthened the coordination and harmonisation across all sectors and stakeholders related to flood risk management. The FD implementation, therefore, is a holistic attempt to outline the needs for action for all sectors related to risk management. The obligation of reviewing the process on a regular basis and to revise the information if needed supports a sustainable approach by discussing the achievements and deficits transparently. The consideration of residual risk (overload and failure of flood defences) and, hence, the incorporation to national legislation is of substantial relevance especially for awareness raising and public information and consultation. Concluding the implementation of the FD is very valuable for strategic planning (mid-term) on national level which is then linked to existing and well-proven mechanisms of detailed planning and funding. The priorities are set on non-structural measures as well as measures fostering flood retention.
\end{abstract}

\section{Setting the Scene}

In the frame of implementing the EU Floods Directive in Austria there are two major aspects to be considered. First, Austria as a federal state has it's competences structured in different levels of administration:

- Federal State: Water law (Floods Directive) and Flood Protection Funding law

- 9 Federal Provinces: Spatial Planning, Building Codes, Emergency Management, etc.

- 95 Districts \& 2100 Municipalities: Zoning, Spatial Development Management, Land Development, Emergency Planning, etc.

\footnotetext{
${ }^{\mathrm{a}}$ Corresponding author: clemens.neuhold@bmlfuw.gv.at
}

Second, there is a high topographical diversity to be considered in developing uniform and comparable methods for the Floods Directive implementation subdivided in
- Alpine
- Pre-Alpine
- Lowland

Due to the varying topographic boundary conditions, Austria is prone to floods with different characteristics, such as:

- high precipitation, high flow velocities, sediment transport and short lead times in Alpine regions

- high vulnerability due to settlements, infrastructure and industry in valleys

- long flood durations in low land areas

- flash floods

- pluvial floods 


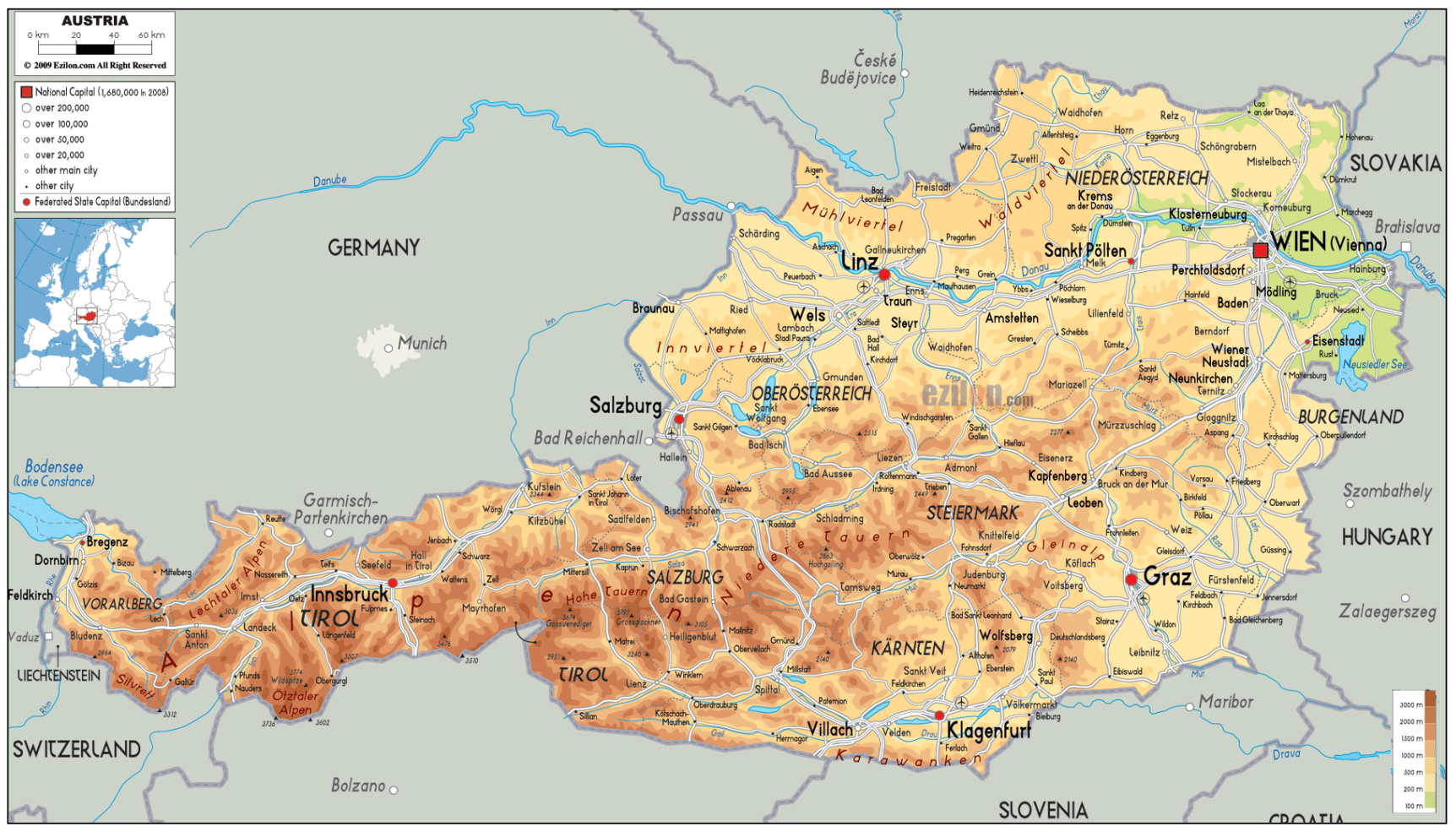

Figure 1. Surface topography of Austria

\section{The directive 2007/60/EC}

Floods have the potential to cause fatalities, displacement of people and damage to the environment, to severely compromise economic development and to undermine the economic activities of the Community.

The EU Directive on the assessment and management of flood risks [2007/60/EC], often referred to as the 'Floods' Directive, was adopted on 23 October 2007. Its aim is to reduce and manage the risks that floods pose to human health, the environment, cultural heritage and economic activity. The approach is based on a six year cycle of planning, subject to the application of transitional arrangements. The development of a Floods Directive was considered after the huge and devastating floods that struck Central Europe in 2002. It came into force with a principal objective to reduce the risk of floods and to take future changes in the risk of flooding as a result of climate change into account. The focus of the FD is broad aiming to reduce the adverse consequences for human health, economic activity as well as the environment and cultural heritage.

The FD is to be implemented in Member States in three stages. During the first stage, the EU Member States should have carried out Preliminary Flood Risk Assessments (PFRAs) for river basins and for coastal zones by 22 December 2011, in order to identify areas of existing or foreseeable future potentially significant flood risk (referred to as 'Areas of Potentially Significant Flood Risk (APSFRs)). An important concept in the FD is flood risk. This is a combination of the probability of the flood occurring and its consequences.
During the second stage, Member States should prepare flood hazard maps and flood risk maps for the APSFRs identified by 22 December 2013. These should identify areas prone to flooding during events with a high (optional), medium and low probability of occurrence, including those where occurrences of floods would be considered an extreme event. The maps will also have to include details of expected flood extent and water depths (flood hazard maps) and economic activities that could be affected, the number of inhabitants at risk and the potential environmental damage (flood risk maps).

The third stage will require Member States to produce catchment-based Flood Risk Management Plans (FRMPs) by 22 December 2015, thereby harmonizing with the WFD River Basin Management Plan (RBMP) cycle. The FRMPs will be focused on prevention, protection and preparedness, setting objectives for managing the flood risk within the APSFRs and setting out a prioritised set of measures for achieving those objectives. Member States should coordinate their flood risk management practice in shared river basins, including with third counties, and shall not undertake measures that would increase the flood risk in neighbouring countries.

Member States should also take into consideration long term developments, including climate change, as well as sustainable land use practices in the flood risk management cycle addressed in the FD. All assessments, maps and plans prepared shall be made available to the public, and Member States are required to encourage the active involvement of interested parties in the preparation of the FRMPs. 
To summarise the FD is designed to:

- establish a framework for the assessment and management of flood risks, aiming at the reduction of the adverse consequences for human health, the environment, cultural heritage and economic activity associated with floods in the Community

- establish a process for producing flood hazard maps and flood risk maps in order to address the flood risk

- in the flood risk management plans address all aspects of flood risk management focusing on prevention, protection, preparedness, including flood forecasts and early warning systems and taking into account the characteristics of the particular river basin or sub-basin.

The FD planning cycle is aligned with that of the WFD and there is a requirement for coordination of the two Directives. It is important to note that, as of October 2013, the first Flood Risk Management Plans have yet to be produced and hence Member States are still undergoing a learning process in how the synergies between the FD and WFD can be taken advantage of at a practical level. (EC, 2014)

\subsection{Coordination and harmonisation in Austria}

To obtaining the national targets such as:

- A comparable, transferable and transparent method for the three steps of implementation

- Defining clear interfaces to existing instruments

- Outlining needs for action and priorities
- Fostering collaboration of all relevant sectors and administrative levels

- Using synergies with the Water Framework Directive

- Informing and consulting the public

and, therefore, ensuring a coordinated planning and implementation under public participation a national "discussion and decision committee (AK-HWRL)" had been established.

This AK-HWRL consists of representatives of different sectors and levels of the administration in Austria. The most influential are:

- The Federal Ministry of Agriculture, Forestry, Environment and Water Management as the Competent Authority and Chair of the AK-HWRL

- The Federal Ministry of Transport, Innovation and Technology

- The Federal Ministry of the Interior

- Federal Province Departments of Water Management, Flood Protection, Spatial Planning, Emergency Management,

- Environmental Agency, Stakeholders, Experts, Consultants, Research, etc.

Due to the Federal System an additional working process had been incorporated to the 3 steps of implementing the FD. The Competent Authority provides for PFRA / APSFR, FHRM and FRMP a "federal blueprint" to the Federal Provinces based on resolutions of the AKHWRL. Adding to this federal blueprint the Federal Provinces provide information, data and knowledge.

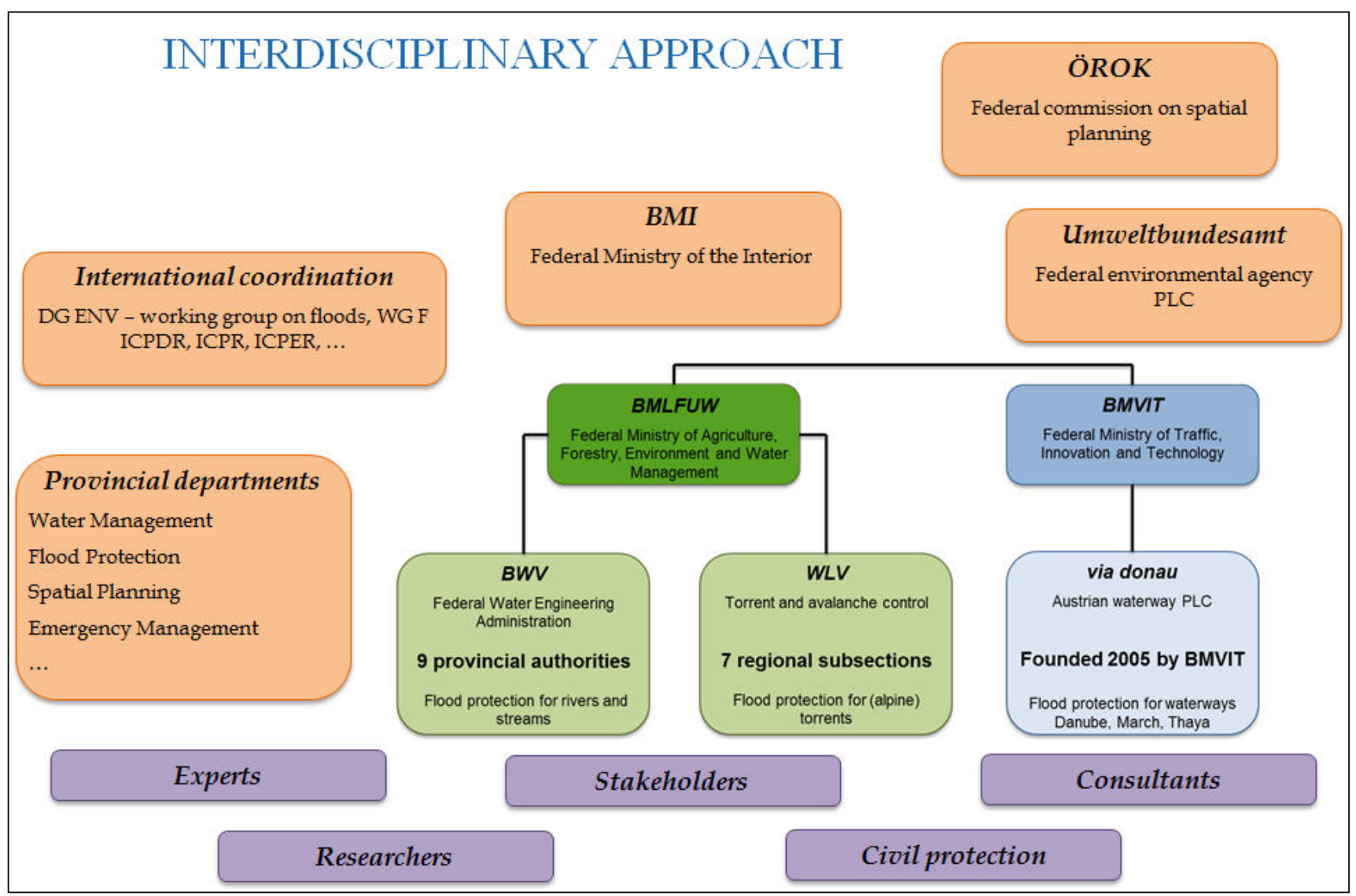

Figure 2. Representatives of different sectors and levels of administration in the frame of the Discussion and Decision Committee AK-HWRL in Austria. 
After that the Competent Authority merges available information and publishes it as a draft. After the information undergoes public information and consultation the implementation steps are revised and published as well as reported to the European Commission.

On international and river basin level (Danube, Rhine and Elbe) coordination and harmonisation had been achieved by the CIS (Common implementation strategy) WG F (working group on floods), chaired by the European Commission and by means of working groups of the International Commissions of the protection of the Danube (ICPDR), Rhine (ICPR) and Elbe (IKSEMKOL).

\subsection{Investments in flood protection in Austria}

Since the 2002 flood event in Austria approximately 2 Billion $€$ had been invested by the Federal State for protection measures (2002-2011).

Complementary investments had been provided by the Federal Provinces and Municipalities. In average the investments in flood protection are 300-400 Mio. $€$ / year.

\section{Results of the Floods Directive implementation in Austria}

In Austria all results and background documents had been made available for the public on the national water information system Austria (http://wisa.bmlfuw.gv.at) and reported to the water information system for Europe (http://water.europa.eu/).

\subsection{PFRA / APSFR in Austria}

Based on a national preliminary flood risk assessment 391 areas of potential significant flood risk had been identified. Referring to boundary conditions such as topography, settled areas, land use development, etc. a broad variability in length has been designated.

The minimum length of APSFR in Austria is $500 \mathrm{~m}$, the maximum length $61,5 \mathrm{~km}$.

\subsection{FHRM in Austria}

For the process of elaborating flood hazard and risk maps focus had been put on addressing uncertainty and reliability of data considered by assigning different shades to inundated areas as well as the consideration and communication of residual risk by overload and failure scenarios.

Flood hazard maps (Fig. 4) had been elaborated on a basin wide scale and published by a web-GIS viewer. Flood risk maps (Fig. 5)had been derived for APSFR only and published by a web-GIS viewer as well.

\section{Gebiete mit potenziellem signifikantem Hochwasserrisiko 22.12.2011}

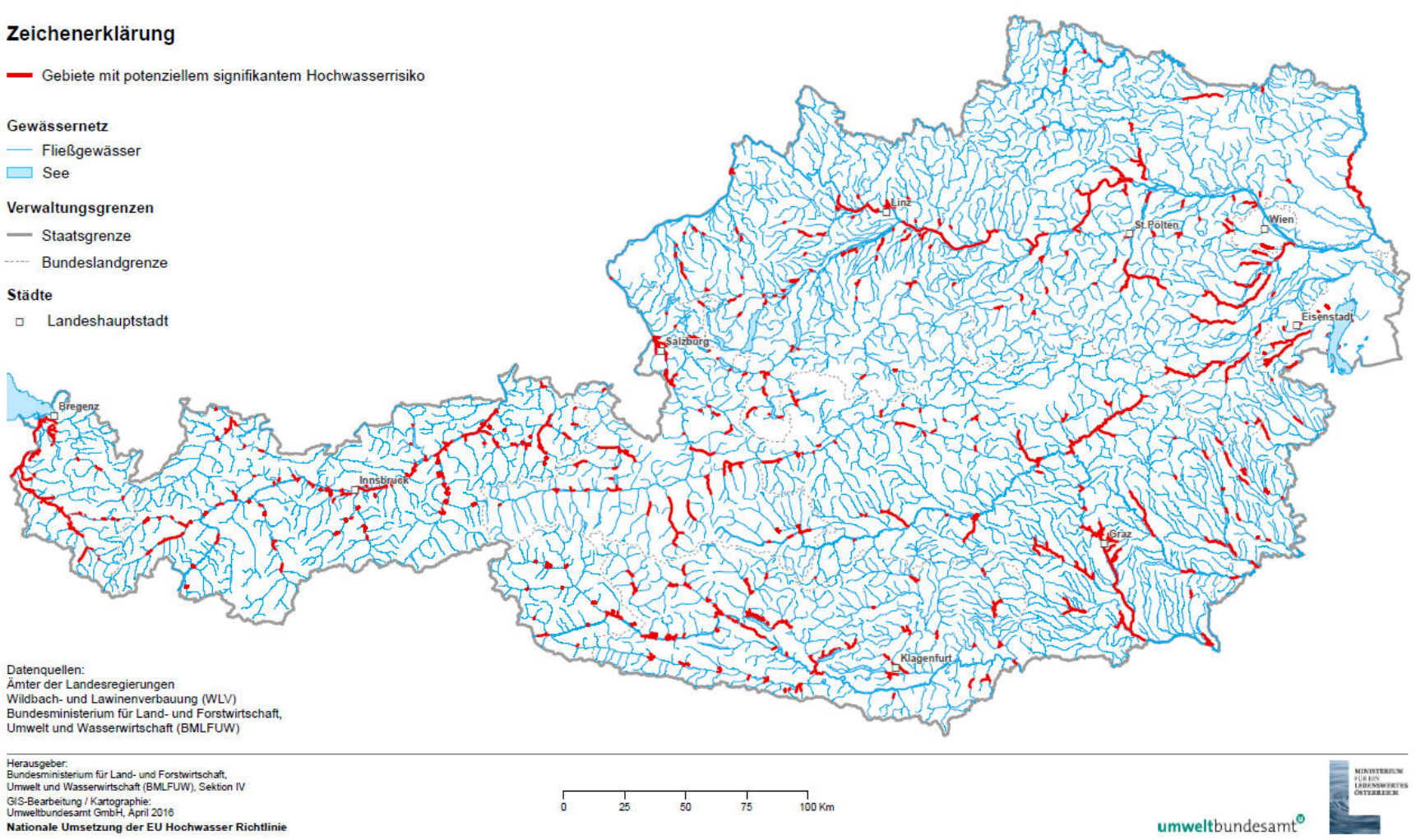

Figure 3. 391 APSFR in Austria 


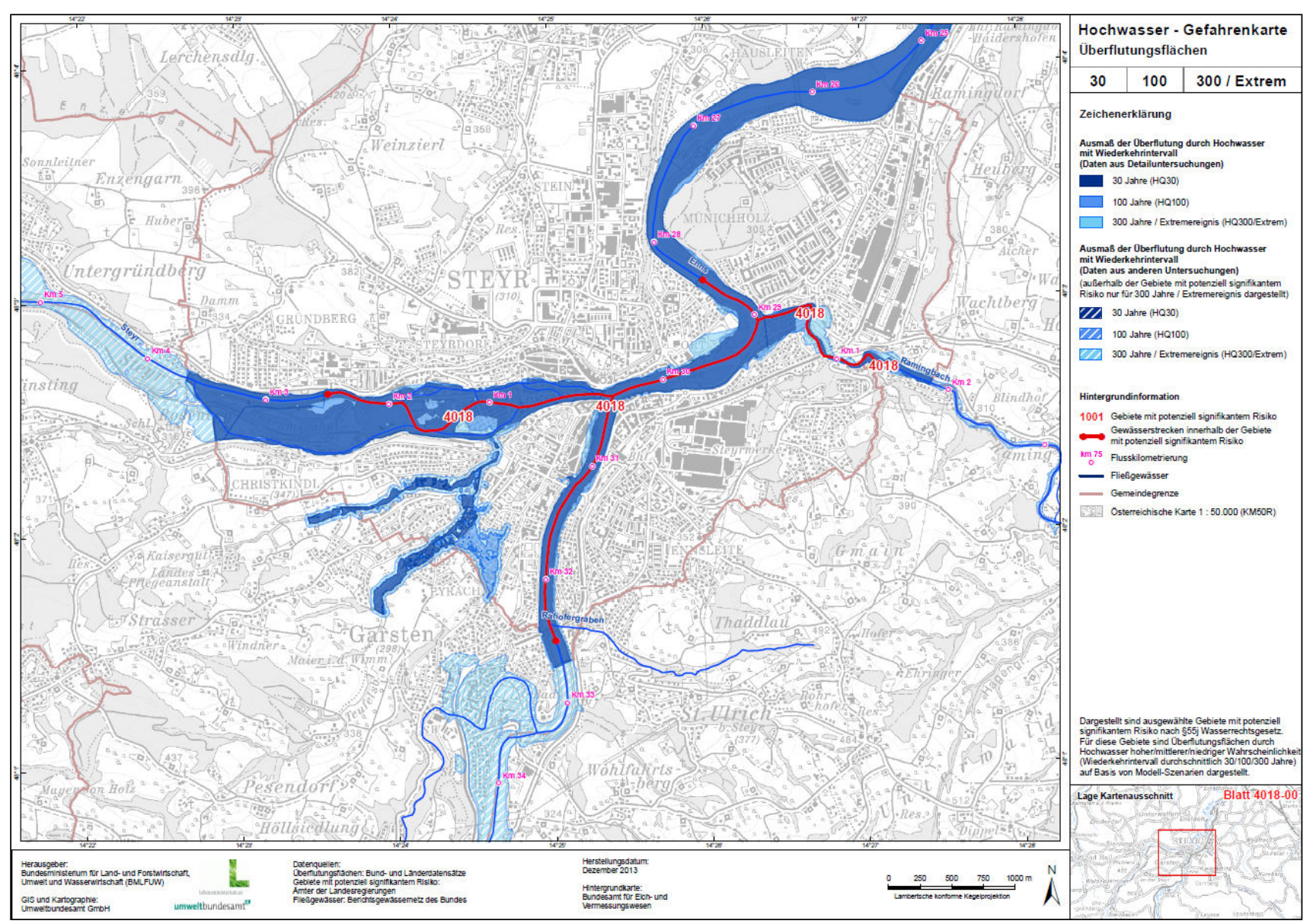

Figure 4. Flood Hazard Map - Inundation Map - in Austria.

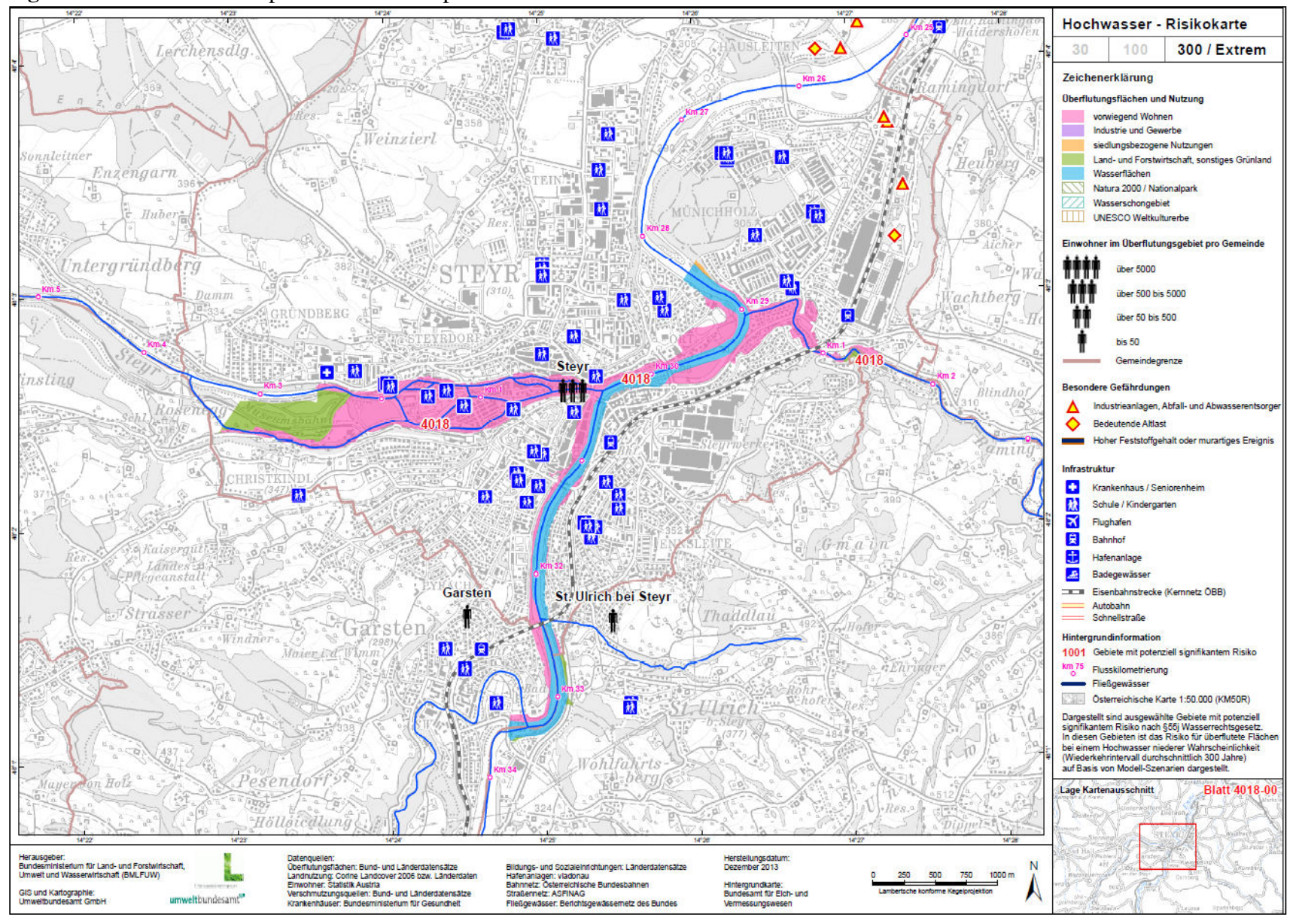

Figure 5. Flood Risk Map in Austria. 




Figure 6. Flood Risk Management Plan in Austria.

\subsection{FRMP in Austria}

In the frame of the national flood risk management plan 4 appropriate objectives had been defined:

- Avoidance of new risks

- Reduction of existing risks

- Strengthening resilience

- Raising awareness

These appropriate objectives shall be achieved by implementing 22 types of measures which had been assigned, if relevant, to the APSFR. The catalogue of measures consists of:

- M01: Compilation and update of hazard zone plans

- M02: Incorporation of hazard zone plans

- M03: Development of concepts, plans, projects, strategies on catchment scale to improve the water and sediment balance

- M04: Compilation and incorporation of local and regional land use planning strategies

- M05: Definition of a framework for implementation and maintenance of flood protection and mitigation measures.

- M06: Improvement of retention capacity on catchment scale

- M07: Restoration of flood plains and sedimentation areas

- M08: Structural protection measures

- M09: Object oriented measures

- M10: Relocation and reallocation

- M11: Improvement of river inspection
- M12: Maintenance of protection and mitigation measures, river maintenance

- M13: Definition of operating instructions for flood prone and flood influencing facilities

- M14: Information of public in an appropriate way

- M15: Improve participation

- M16: Educational activities

- M17: Implementation of monitoring, forecasting, warning systems

- M18: Compilation of emergency plans

- M19: Ensure availability of facilities for emergency

- M20: Emergency response

- M21: Evaluation and repair of damages

- M22: Documentation and analysis

Based on the need for action, measure characteristics (non-structural, climate change adaptation, contribution to WFD targets) and the state of implementation priorities had been set. In principle priorities are:

- high priority on reliable data and planning

- medium priority on non-structural / ecologically oriented measures

- low priority on technical / structural measures

Flood risk management plans had been developed for the level A for the Rivers Danube, Rhine and Elbe, on National Level and on APSFR level. Background documents, catalogue of measures, guidelines, etc. had been made available and published. 


\subsection{Links between FD and WFD in Austria}

Due to the same timeline of implementation as well as having the same competent authority in Austria the implementations of both directives are well coordinated. There are numerous joint activities, and projects conducted.

Further, there is an extensive experience in implementing nature oriented flood protection and mitigation measures (e.g. natural water retention measures) with potential multiple benefits. The links and coordination between these two sectors are also referred to in the frame of funding priorities and planning principles which are:

- non-structural measures have priority over structural measures

- measures in the catchment area have priority over measures along the main channel

- retention measures have priority over linear structural measures

- natural methods of building have priority over technical methods

\section{Experience and lessons learnt}

The implementation of the EU Floods Directive in Austria clearly strengthened the coordination and harmonisation across sectors, administrational levels and stakeholders.

The Flood Risk Management Plan is a holistic attempt to outline the needs for action for all sectors such as water management, flood protection, spatial planning, emergency planning, etc.

The obligation to review the process and to revise relevant information in the frame of a cyclic work flow supports a sustainable approach by discussing achievements and deficits transparently.

The Consideration of residual risk (e.g. due to overload and failure) is of substantial relevance especially for awareness raising and public information and consultation.

\section{Conclusions}

The implementation of the EU Floods Directive is very valuable for strategic planning on national level.

Clear links to existing and well-proven mechanisms of detailed planning and funding are essential.

Priorities in Austria are set on reliable planning, on non-structural measures and on measures fostering retention.

\section{References}

European Commission (2014): Links between the Floods Directive (2007/60/EC) and the Water Framework Directive (2000/60/EC). Resource Document. Technical Report 2014078. 